\title{
THE OUTER OBLIQUE BOUNDARY PROBLEM OF POTENTIAL THEORY
}

\author{
MARTIN GROTHAUS AND THOMAS RASKOP
}

\begin{abstract}
In this article we prove existence and uniqueness results for solutions to the outer oblique boundary problem for the Poisson equation under very weak assumptions on boundary, coefficients and inhomogeneities. Main tools are the Kelvin transformation and the solution operator for the regular inner problem, provided in [1]. Moreover we prove regularisation results for the weak solutions of both, the inner and the outer problem. We investigate the non-admissible direction for the oblique vector field, state results with stochastic inhomogeneities and provide a Ritz-Galerkin approximation. The results are applicable to problems from Geomathematics, see e.g. [2] and [3].
\end{abstract}

Key words and phrases: Kelvin transformation; Oblique boundary problem; Poisson equation; Sobolev spaces; Submanifolds; Weak solutions.

2000 Mathematics Subject Classification: 31B20, 35J05, 35J25, 46E35, 32C25, 86A30.

\section{INTRODUCTION}

The main subject of the present article are existence results for solutions to the outer oblique boundary problem for the Poisson equation. It is based on the article [1], in which a theory for deterministic as well as stochastic inhomogeneities and solutions to the regular inner problem is provided. The problem is called outer problem because it is defined on an outer domain $\Sigma \subset \mathbb{R}^{n}$. This is a domain $\Sigma$, having the representation $\Sigma=\mathbb{R}^{n} \backslash \bar{D}$ where $0 \in D$ is a bounded domain. Consequently, $\partial \Sigma$ divides the euclidean space $\mathbb{R}^{n}$ into a bounded domain $D$, called inner domain, and an unbounded domain $\Sigma$, called outer domain. The problem defined on $D$ and $\partial D$ is called inner problem. The Poisson equation on $\Sigma$ is given by

$$
\Delta u=f
$$

and the oblique boundary condition by

$$
\langle\underline{a} \cdot \nabla u\rangle+b u=g,
$$

on $\partial \Sigma$. This condition is called regular if the equation

$$
|\langle\underline{a} \cdot \nu\rangle|>C>0,
$$

holds on $\partial \Sigma$ for a constant $0<C<\infty$. A classical solution corresponding to continuous $\underline{a}, b, g$ and $f$ of the outer oblique boundary problem for the Poisson equation is a function $u \in C^{2}(\Sigma) \cap C^{1}(\bar{\Sigma})$ fulfilling the first two equations and having the property that $u \in \mathcal{O}\left(\frac{1}{|x|}\right)$ for $|x|$ tending to infinity. In order to allow very weak assumptions on boundary, coefficients and inhomogeneities, we are interested in weak solutions to this 
problem. In [1] an existence and uniqueness result for the weak solution to the inner oblique boundary problem is presented. To be more precisely, if $\partial \Sigma$ is a $C^{1,1}$-boundary of a bounded domain $\Sigma,|\langle\underline{a} \cdot \nu\rangle|>C>0$ on $\partial \Sigma, \frac{\underline{a}}{\langle\underline{a} \cdot \nu\rangle}-\nu \in H^{1, \infty}\left(\partial \Sigma ; \mathbb{R}^{n}\right), \frac{b}{\langle\underline{a} \cdot \nu\rangle} \in L^{\infty}(\partial \Sigma)$ and $\operatorname{ess} \inf _{\partial \Sigma}\left(\frac{b}{\langle\underline{a} \cdot \nu\rangle}-\frac{1}{2} \operatorname{div}_{\partial \Sigma}\left(\frac{a}{\langle\underline{a} \cdot \nu\rangle}-\nu\right)\right)>0$, then for every $\frac{g}{\langle\underline{a} \cdot \nu\rangle} \in H^{-\frac{1}{2}, 2}(\partial \Sigma)$ and $f \in\left(H^{1,2}(\Sigma)\right)^{\prime}$ there exists one and only one $u \in H^{1,2}(\Sigma)$ fulfilling the weak formulation. Additionally the solution depends continuously on the data. Beside from some results for Sobolev spaces defined on sub manifolds, the crucial point in the proof is to show coercivity of the bilinear form related to the weak formulation. Therefore we use a Poincaré inequality, namely

$$
\int_{\Sigma}\langle\nabla u \cdot \nabla u\rangle d \lambda^{n}+\int_{\partial \Sigma} u^{2} d H^{n-1} \geq C\left(\int_{\Sigma} u^{2} d \lambda^{n}+\int_{\Sigma}\langle\nabla u \cdot \nabla u\rangle d \lambda^{n}\right),
$$

for all $u \in H^{1,2}(\Sigma)$. For details see the reference given above. Important is, that this Poincaré inequality is only available for bounded domains, so we can not apply the same techniques to the outer setting. Before we go to the outer problem we prove a regularisation result for the inner problem. This well be used later on in order to prove the main results of this article. If $\partial \Sigma$ is a $C^{2,1}$-boundary of a bounded domain $\Sigma$, $\frac{\underline{a}}{\langle\underline{a} \cdot \nu\rangle}-\nu \in H^{2, \infty}\left(\partial \Sigma ; \mathbb{R}^{n}\right), \frac{b}{\langle\underline{a} \cdot \nu\rangle} \in H^{1, \infty}(\partial \Sigma), \frac{g}{\langle\underline{a} \cdot \nu\rangle} \in H^{\frac{1}{2}, 2}(\partial \Sigma)$ and $f \in L^{2}(\Sigma)$, we are able to show that the weak solution is even a strong solution, i.e. $u \in H^{2,2}(\Sigma)$. In the proof we show that the weak solution fulfills the requirements of a regularisation result for the weak Neumann problem.

Then we tackle the outer problem. Our approach in order to provide a weak solution is transforming this problem to a corresponding inner problem, using the Kelvin transformation. This transformation defines for each outer domain $\Sigma$ an inner domain $\Sigma^{K}$ via

$$
\Sigma^{K}:=\left\{\frac{x}{|x|^{2}} \mid x \in \Sigma\right\} \cup\{0\} .
$$

In turn, we get for each function $u$ defined on $\Sigma^{K}$ a function $v$ by

$$
v(x):=\frac{1}{|x|^{n-2}} u\left(\frac{x}{|x|^{2}}\right),
$$

for all $x \in \Sigma$. The first transformation leaves the regularity of $\partial \Sigma$ invariant, while the second has the important property

$$
\Delta v(x)=\frac{1}{|x|^{n+2}}(\Delta u)\left(\frac{x}{|x|^{2}}\right), \quad x \in \Sigma,
$$

for all $u \in C^{2}\left(\Sigma^{K}\right)$. Our idea is to use this transformations in order to provide a weak solution. We transform the outer problem into a corresponding inner problem, then solve this problem and finally transform the weak solution of the inner problem to a function defined in the outer domain. The transformations $T_{1}(f)$ and $T_{2}(g)$ of the inhomogeneities as well as $T_{3}(\underline{a})$ and $T_{4}(b)$ of the coefficients can be identified by standard calculus. The problem is to find the right function spaces and then to extend the transformations to these spaces. In order to identify them we have to take care of two main aspects. First, 
the image spaces under the transformations have to fulfill the requirements of the existence and uniqueness result for the regular inner problem. Otherwise we cannot apply the solution operator for the inner problem. Second, the transformations should be continuous. Otherwise the weak solution of the outer problem will not depend continuously on the inhomogeneities. We are able to show that the spaces $H^{-\frac{1}{2}, 2}(\partial \Sigma)$ for the boundary inhomogeneity, $\left(H_{|x|^{2},|x|^{3}}^{1,2}(\Sigma)\right)^{\prime}$ for the domain inhomogeneity and $H_{\frac{1}{|x|^{2}}, \frac{1}{|x|}}^{1,2}(\Sigma)$ for the weak solution, are a suitable choice. Here we have Sobolev spaces equipped with weighted Lebesgue measures. Under this conditions we are able to prove that if $\Sigma$ is an outer $C^{1,1}$ domain, $\underline{a} \in H^{1, \infty}\left(\partial \Sigma ; \mathbb{R}^{n}\right)$ and $b \in L^{\infty}(\partial \Sigma)$, fulfilling $\left|\left\langle\left(T_{3}(\underline{a})\right)(y) \cdot \nu^{K}(y)\right\rangle\right|>C>0$ and ess $\inf _{\partial \Sigma^{K}}\left\{\frac{T_{4}(b)}{\left\langle T_{3}(\underline{a}) \cdot \nu^{K}\right\rangle}-\frac{1}{2} \operatorname{div}_{\partial \Sigma^{K}}\left(\frac{T_{3}(\underline{a})}{\left\langle T_{3}(\underline{a}) \cdot \nu^{K}\right\rangle}-\nu^{K}\right)\right\}>0$, for each $g \in H^{-\frac{1}{2}, 2}(\partial \Sigma)$ and $f \in\left(H_{|x|^{2},|x|^{3}}^{1,2}(\Sigma)\right)^{\prime}$, there exists $u:=K\left(S_{T_{3}\left(\underline{a}, T_{4}(b)\right.}^{\text {in }}\left(T_{1}(f), T_{2}(g)\right)\right)$ with $u \in H_{\frac{1}{|x|^{2}}, \frac{1}{|x|}}^{1,2}(\Sigma)$ fulfilling the continuity estimate

$$
\|u\|_{H_{\frac{1}{|x|^{2}, \frac{1}{|x|}}(\Sigma)}^{1,2}} \leq C\left(\|f\|_{\left(H_{|x|^{2},|x|^{3}}^{1,2}(\Sigma)\right)^{\prime}}+\|g\|_{H^{-\frac{1}{2}, 2}(\partial \Sigma)}\right) .
$$

Furthermore we can show that if $\Sigma$ is an outer $C^{2,1}$-domain and $\underline{a} \in H^{2, \infty}\left(\partial \Sigma ; \mathbb{R}^{n}\right)$ and $b \in H^{1, \infty}(\partial \Sigma), f \in L_{|x|^{2}}^{2}(\Sigma)$ and $g \in H^{\frac{1}{2}, 2}(\partial \Sigma)$ we have $u \in H_{\frac{1}{|x|^{2}}, \frac{1}{|x|}, 1}^{2,2}(\Sigma)$ for the weak solution to the outer problem. Additionally, it fulfills the classical formulation almost everywhere and a corresponding continuity estimate holds. Because of the Kelvin transformation we get a transformed non admissible direction for the oblique vector field $\underline{a}$. For $\mathbb{R}^{2}$ we can explicitly calculate this direction. It only depends on the geometry of the surface $\partial \Sigma$.

This article is organized as follows. In Section 2 we define the function spaces we will use. This are mainly the spaces of smooth functions and spaces of weakly differentiable functions. We introduce Sobolev spaces on sub manifolds and Sobolev spaces equipped with weighted Lebesgue measures. Closing the section, we present some important results about these spaces. In Section 3 we present the weak theory for the regular inner problem including an existence and uniqueness result for a broad class of inhomogeneities. This are mainly the results contained in [1], except of the regularisation result at the end of the section. In Section 4 we start with the investigation of the outer problem. We introduce transformations which will be used in order to transform the outer problem to a corresponding inner problem. Also some important properties of those transformations are proved. They will be important in Section 5. Here we state the outer problem and in the following we will be able to prove the existence of a weak solution for a very general class of inhomogeneities. The modified condition which occurs because of the Kelvin transformation is investigated in a separate subsection at the end of Section 5. Finally, we state a remark about stochastic inhomogeneities and a Ritz-Galerkin approximation method. Both can be implemented, using the techniques from [1].

Our analysis of the outer problem is motivated by problems from Geomathematics. Here 
oblique boundary problems arise frequently, because in general the normal of the Earth's surface does not coincide with the direction of the gravity vector. Therefore, the oblique boundary condition is more suitable then a Neumann boundary condition. For details see [3] or [4].

The main progress achieved in this article can be summarized by the following core results:

- A regularisation result for a strong solution to the inner problem, i.e. $u \in$ $H^{2,2}(\Sigma)$, is proved, see Theorem 3.6.

- The transformation of the outer oblique boundary problem for the Poisson equation to a corresponding inner problem is provided. Important properties of this transformation are proved, see Lemmata 4.7, 4.11, 4.13 and 4.14.

- The existence of a weak solution to the outer problem under weak assumptions on coefficients and surface for a large class of inhomogeneities is proved, see Theorem 5.2 .

- An existence result for a strong solution under additional regularity assumptions is proved and the connection to the classical problem is established, see Theorem 5.4 .

- The transformed condition on the oblique vector field is investigated, see Subsection 5.2.

- Stochastic inhomogeneities as well as an existence result for the stochastic weak solution can be implemented, see Remark 5.5 (i). Additionally a Ritz-Galerkin approximation method for numerical computations is available, see Remark 5.5 (ii). For details about these results we refer to [1]. The results are applicable to problems from Geomathematics, see e.g. [2] and [3].

Martin Grothaus and Thomas Raskop, Mathematics Department,

University of Kaiserslautern, P.O.Box 3049, 67653 Kaiserslautern, Germany.

E-MAIL: GROTHAUS@MATHEMATIK. UNI-KL.DE 